\title{
Primary Splenic Lymphoma, a Rare yet Possible Diagnosis: A Case Report and Review of the Literature
}

\author{
Parima Safe (iD ${ }^{1,}{ }^{*}$, Mohammad Ali Moradi Ghadi ${ }^{2}$, Athena Alipour Faz ${ }^{1}$ and Hassan Peyvandi (iD ${ }^{3,}{ }^{* *}$ \\ ${ }^{1}$ Researcher at Clinical Research Center of Loghman Hakim Hospital, Shahid Beheshti University of Medical Sciences, Tehran, Iran \\ ${ }^{2}$ Loghman Hakim Hospital, Shahid Beheshti University of Medical Sciences, Tehran, Iran \\ ${ }^{3}$ Hearing Disorders Research Center, Shahid Beheshti University of Medical Sciences, Tehran, Iran \\ Corresponding author: Researcher at Clinical Research Center of Loghman Hakim Hospital, Shahid Beheshti University of Medical Sciences, 1333635445, Tehran, Iran. Tel: \\ +98-2151025153, Email: parimasafe@gmail.com \\ "Corresponding author: Associate Professor of Surgery, Hearing Disorders Research Center, Shahid Beheshti University of Medical Sciences, 1333635445, Tehran, Iran. Tel: \\ +98-2151025153, Email: hassan.peyvandi@gmail.com
}

Received 2019 July 20; Revised 2019 August 11; Accepted 2019 August 13.

\begin{abstract}
Introduction: The spleen is subject to a variety of benign and malignant disorders, which can be manifested by nonspecific symptoms or signs. Hence, the diagnosis is often made based on radiological examinations and histopathological studies.

Case Presentation: Herein we present a case report of a patient with pathologically approved primary splenic lymphoma (PSL). The patient was a 63-year-old woman presented with abdominal pain, predominantly in the left upper quadrant, since 1 month. Complete blood count (CBC) was normal except for mild anemia. Liver enzyme studies showed an elevated alkaline phosphatase level. Ultrasound (US) depicted a huge hypoechoic splenic mass and computed tomography (CT) confirmed the lesion to be a hypodense mass partially exophytic from the superior aspect of the spleen measuring up to $124 \mathrm{~mm}$ in size. The patient underwent a midline laparotomy and splenectomy and the spleen was sent for histopathological examination, which revealed high-grade B-cell lymphoma measuring $17 \times 16 \times 10 \mathrm{~cm}$ in size with capsular invasion and without any lymphovascular invasion.

Conclusions: This case report highlights the importance of considering splenic lymphoma as a differential diagnosis, although rare, in a patient with abdominal pain and nonspecific clinical findings.
\end{abstract}

Keywords: Abdominal Pain, Case Report, High-Grade B-Cell Lymphoma, Spleen, Splenectomy

\section{Introduction}

The spleen is infrequently involved in neoplastic processes. Primary benign splenic tumors include lymphangioma, hemangioma, littoral cell angioma and splenic cyst, and solid lesions such as inflammatory pseudotumor and hamartoma. Lymphoma and angiosarcoma are primary malignant neoplasms affecting the spleen $(1,2)$. Primary splenic lymphoma (PSL), which is usually of nonHodgkin B-cell origin, comprises less than $1 \%$ of all lymphomas $(2,3)$. Due to rarity and unspecified presentations such as fever, malaise, weight loss, lower upper quadrant pain, or discomfort due to the enlarged spleen $(4,5)$, the diagnosis of splenic lymphoma is often hindered (5), which can potentially influence its prognosis. The physicians must be aware of clinical and imaging features of malignant as well as benign splenic pathologies to be able to differentiate them when encountered symptomatically or incidentally (6).

\section{Case Presentation}

A 63-year-old female with a 1-month history of abdominal pain presented to our emergency department. The pain was more intense in the left upper quadrant with a colicky pattern, which got worse after eating meals and was not positional. The patient complained of weakness, fatigue, fever, chills, and night sweats, as well as weight loss of about $5 \mathrm{~kg}$ in 1 month. She also reported constipation and mild dyspnea but did not experience nausea, vomiting, urinary symptoms, or jaundice. The medical history of the patient was clear and surgical history was positive for laparoscopic cholecystectomy. She denied any drug or substance abuse and the family history was negative. Physical examinations revealed the scar of cholecystectomy, mild left upper quadrant abdominal tenderness without rebound tenderness or muscle guarding, and splenomegaly. No lymphadenopathy was detected. The rest of the examination including rectal evaluations was unremarkable. Complete blood count (CBC) showed mild 
anemia (hemoglobin: $10.6 \mathrm{~g} / \mathrm{dL}$ ). Other laboratory blood tests were within normal ranges, except for an elevated alkaline phosphatase level (620 U/L). An abdominal ultrasound (US) was done, showing a huge hypoechoic mass in the superior aspect of the spleen, measuring $90 \times 88 \mathrm{~mm}$ in size. Computed tomography (CT) scan displayed a hypodense mass partially exophytic from the superior aspect of the spleen, measuring approximately $124 \times 94 \times 104 \mathrm{~mm}$ in size (Figure 1). No lymphadenopathy was identified in the abdominal and pelvic region. The patient underwent a midline laparotomy and splenectomy and the spleen (Figure 2) was sent for histopathological examination, which revealed a high-grade B-cell lymphoma measuring $17 \times 16$ $\times 10 \mathrm{~cm}$ in size with capsular invasion without lymphovascular invasion. The patient had an uneventful postoperative course and was referred to an oncologist to complete the treatment process.

\section{Discussion}

Primary neoplasms of the spleen can be divided into vascular neoplasms originating from the red pulp, and lymphoid neoplasms arising from the white pulp. Hemangioma, lymphangioma, and hamartoma are among primary vascular neoplasms of the spleen (2). Non-Hodgkin's lymphoma (NHL) is the most common hematological malignancy of the spleen, with diffuse large B-cell lymphoma being the most common type. About 30\% to $40 \%$ of NHL and one-third of all Hodgkin's lymphoma involve the spleen at presentation (7). PSL is characterized as limited involvement of the spleen and immediately nearby lymph nodes, whereas the secondary type, which is more common, is defined as lymphomatous involvement of the spleen, as well as the nodes, other than splenic hilar ones (8).

Diagnosing PSL can be challenging since it often has nonspecific manifestations and can even present with complications such as splenic rupture and hypersplenism $(3,4)$. In a retrospective study conducted by Bairey et al. (9) on 87 patients diagnosed with primary splenic diffuse large B-cell lymphoma, the patients had a mean age of 59.6 years and $57.5 \%$ were male; $97 \%$ of them presented with splenic masses, $84 \%$ with elevated lactate dehydrogenase levels, $84 \%$ with splenomegaly, $81 \%$ with abdominal pain, and 59\% with B symptoms. In 39 patients, splenectomy and in 46 patients, core-needle biopsy led to the diagnosis. They also came to the conclusion that splenectomy at diagnosis can improve survival, particularly at an early stage. Chen et al. (10) analyzed 53 cases of splenic lymphoid and hematopoietic tissue tumors and concluded that splenectomy can yield a partial or complete improvement of blood counts. According to a study carried out by Djokic et al.
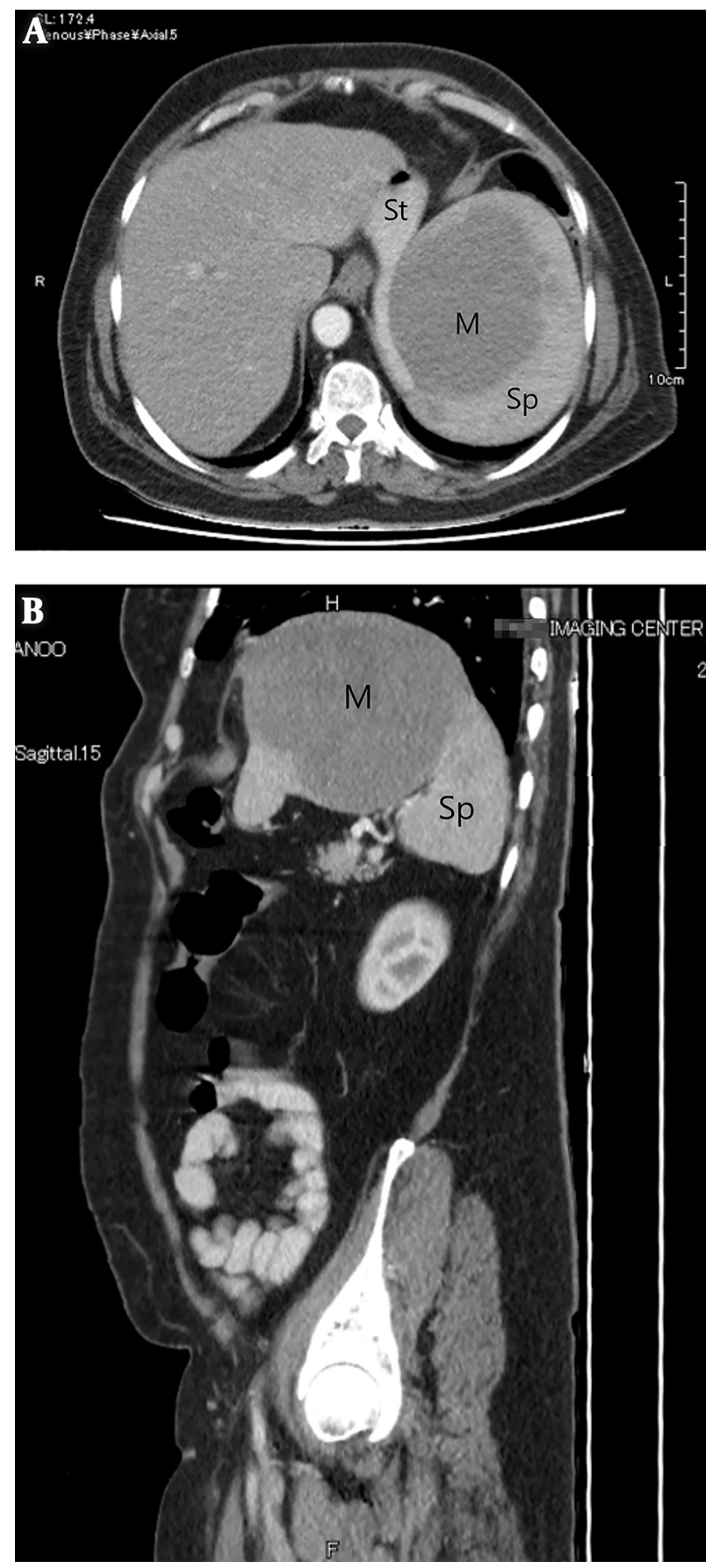

Figure 1. Axial (A) and sagittal(B) section of the abdominal CT scan demonstrating hypodense mass (M) partially exophytic from the superior aspect of the spleen ( $\mathrm{Sp}$ ) with mass effect on the stomach (St)

(11) splenomegaly accompanied by malaise and weight loss should raise suspicion for underlying neoplastic condition, as seen in our patient, and would require further di- 


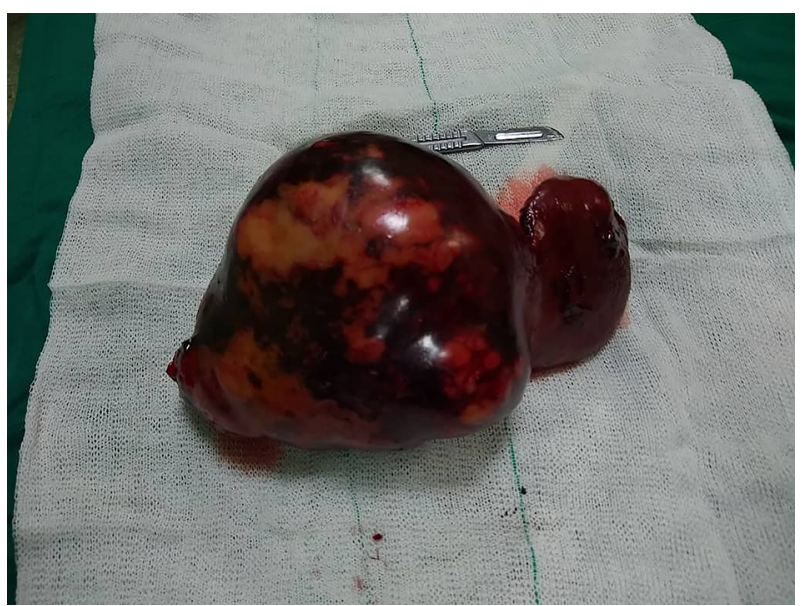

Figure 2. Gross view of the resected spleen and the accompanying mass

agnostic approaches.

A number of splenic lymphoma cases have been reported in the literature: Kattepur et al. (12) presented a 50year-old female patient without comorbidities, who presented with left upper quadrant abdominal pain since 2 months and a scenario similar to that of our patient's. Ingle and Ingle (13) reported a female patient with abdominal pain and weight loss without fever or lymphadenopathy. Other than being febrile, our patient shared similar symptoms to those experienced by the 41-year-old patient. Khalid et al. (14) described a 68-year-old male patient with left-sided abdominal pain, massive splenomegaly, and thrombocytopenia.

Navarro et al. (15) reported a 69-year-old female patient with abdominal pain and large splenomegaly diagnosed with splenic lymphoma, who unfortunately died due to septic shock 4 months after diagnosis.

\subsection{Conclusions}

Rarity and the unspecified presentation should not cause physicians to dismiss splenic lymphoma from the differential diagnoses. When diagnosed at an early stage, PSL can carry a better prognosis and be managed more prosperously.

\section{Acknowledgments}

None to declare.

\section{Footnotes}

Author's Contribution: Parima Safe, Mohammad Ali Moradi Ghadi, Athena Alipour Faz and Hassan Peyvandi contributed equally in preparing the manuscript and are accountable for all aspects of the work.

Conflict of Interests: The authors declare that they have no conflict of interest.

Financial Disclosure: The authors have no financial disclosure.

Funding/Support: None to declare.

Patient's Consent: A written informed consent was obtained from the patient and she agreed that the physicians could publish her disease-related article and any accompanying images with personal information was deleted.

\section{References}

1. Giovagnoni A, Giorgi C, Goteri G. Tumours of the spleen. Cancer Imaging. 2005;5:73-7. doi: 10.1102/1470-7330.2005.0002. [PubMed: 16154823]. [PubMed Central: PMC1665244].

2. Kaza RK, Azar S, Al-Hawary MM, Francis IR. Primary and secondary neoplasms of the spleen. Cancer Imaging. 2010;10:173-82. doi: 10.1102/14707330.2010.0026. [PubMed: 20713317]. [PubMed Central: PMC2943678].

3. Spier CM, Kjeldsberg CR, Eyre HJ, Behm FG. Malignant lymphoma with primary presentation in the spleen. A study of 20 patients. Arch Pathol Lab Med.1985;109(12):1076-80. [PubMed: 3840980].

4. Ingle SB, Hinge Ingle CR. Primary splenic lymphoma: Current diagnostic trends. World J Clin Cases. 2016;4(12):385-9. doi: 10.12998/wjcc.v4.i12.385. [PubMed: 28035311]. [PubMed Central: PMC5156875].

5. Wu CM, Cheng LC, Lo GH, Lai KH, Cheng CL, Pan WC. Malignant lymphoma of spleen presenting as acute pancreatitis: A case report. World J Gastroenterol. 2007;13(27):3773-5. doi: 10.3748/wjg.v13.i27.3773. [PubMed: 17659747]. [PubMed Central: PMC4250659].

6. Yazici P, Aydin U, Ersin S, Kaplan H. Hamartoma - a rare benign tumor of the spleen: A report of four cases. Eurasian J Med. 2008;40(1):48-51. [PubMed: 25610025]. [PubMed Central: PMC4261320].

7. Saboo SS, Krajewski KM, O’Regan KN, Giardino A, Brown JR, Ramaiya $\mathrm{N}$, et al. Spleen in haematological malignancies: Spectrum of imaging findings. BrJ Radiol.2012;85(1009):81-92. doi:10.1259/bjr/31542964. [PubMed: 22096219]. [PubMed Central: PMC3473934].

8. Leite NP, Kased N, Hanna RF, Brown MA, Pereira JM, Cunha R, et al. Cross-sectional imaging of extranodal involvement in abdominopelvic lymphoproliferative malignancies. Radiographics. 2007;27(6):1613-34. doi:10.1148/rg.276065170. [PubMed:18025507].

9. Bairey O, Shvidel L, Perry C, Dann EJ, Ruchlemer R, Tadmor T, et al. Characteristics of primary splenic diffuse large B-cell lymphoma and role of splenectomy in improving survival. Cancer. 2015;121(17):290916. doi: 10.1002/cncr.29487. [PubMed: 26096161].

10. Chen DB, Shen DH, Zhang H, Wang Y, Song QJ, Yang SM, et al. [Tumors of lymphoid and hematopoietic tissue of spleen: A clinicopathologic analysis of 53 cases]. Zhonghua Bing Li Xue Za Zhi. 2017;46(11):77581. Chinese. doi: 10.3760/cma.j.issn.0529-5807.2017.11.008. [PubMed: 29136691].

11. Djokic M, Plesnik B, Petric M, Trotovsek B. Massive splenomegaly due to B-cell lymphoma: A case report. Int J Surg Case Rep. 2018;48:76-8. doi: 10.1016/j.ijscr.2018.05.013. [PubMed: 29885916]. [PubMed Central: PMC6041427].

12. Kattepur AK, Rohith S, Shivaswamy BS, Babu R, Santhosh CS. Primary splenic lymphoma: A case report. Indian J Surg Oncol. 2013;4(3):287-90. doi: 10.1007/s13193-013-0243-x. [PubMed: 24426740]. [PubMed Central: PMC3771062]. 
13. Ingle $\mathrm{SB}$, Ingle $\mathrm{CR}$. Splenic lymphoma with massive splenomegaly: Case report with review of literature. World J Clin Cases. 2014;2(9):47881. doi: 10.12998/wjcc.v2.i9478. [PubMed: 25232555]. [PubMed Central: PMC4163774].

14. Khalid S, Daw JH, Daw H, Haddad A. Primary splenic diffuse large Bcell lymphoma: A rare case of massive splenomegaly and thrombocy- topenia. Cureus. 2018;10(7). e3026. doi:10.7759/cureus.3026. [PubMed: 30254815]. [PubMed Central: PMC6150744].

15. Navarro JT, Ribera JM, Perez-Piteira J, Vaquero M, Zarco MA, Junca J, et al. [Lymphoma of the marginal zone of the spleen. A case study]. Med Clin (Barc). 1996;106(4):141-3. Spanish. [PubMed: 8948931]. 